\title{
Brain markers predicting response to cognitive- behavioral therapy for social anxiety disorder: an independent replication of Whitfield-Gabrieli et al. 2015
}

\author{
Yoni K. Ashar (1)', Joseph Clark², Faith M. Gunning (1)', Philippe Goldin ${ }^{3}$, James J. Gross ${ }^{4}$ and Tor D. Wager (1) 5
}

\begin{abstract}
Predictive brain markers promise a number of important scientific, clinical, and societal applications. Over 600 predictive brain markers have been described in published reports, but very few have been tested in independent replication attempts. Here, we conducted an independent replication of a previously published marker predicting treatment response to cognitive-behavioral therapy for social anxiety disorder from patterns of resting-state fMRI amygdala connectivity'. The replication attempt was conducted in an existing dataset similar to the dataset used in the original report, by a team of independent investigators in consultation with the original authors. The precise model described in the original report positively predicted treatment outcomes in the replication dataset, but with marginal statistical significance, permutation test $p=0.1$. The effect size was substantially smaller in the replication dataset, with the model explaining $2 \%$ of the variance in treatment outcomes, as compared to $21 \%$ in the original report. Several lines of evidence, including the current replication attempt, suggest that features of amygdala function or structure may be able to predict treatment response in anxiety disorders. However, predictive models that explain a substantial amount of variance in independent datasets will be needed for scientific and clinical applications.
\end{abstract}

Predictive brain markers promise a number of important scientific, clinical, and societal applications ${ }^{2}$. Yet, success in this domain will depend on the replicability and generalizability of brain marker predictions ${ }^{3-6}$. Replicability is at the foundation of scientific enterprise, and it has recently become a subject of increased attention in a number of fields including psychology $y^{7,8}$, translational neuroscience ${ }^{9,10}$, medicine $e^{11,12}$, and more ${ }^{13-16}$. This focus has generated a growing awareness that many published findings cannot be replicated, along with a move towards methods promoting replicability ${ }^{17-21}$.

\footnotetext{
Correspondence: Tor D. Wager (tor.d.wager@dartmouth.edu)

'Department of Psychiatry, Weill Cornell Medicine, New York, NY, USA

2Department of Psychology and Neuroscience, University of Colorado Boulder, Boulder, CO, USA
}

Full list of author information is available at the end of the article
The replicability of most brain markers has not been assessed. A recent review found that of $\sim 450$ published predictive brain markers, only $\sim 10 \%$ have been tested on independent data, and only two clinical markers (one for Alzheimer's disease and one for Parkinson's disease) have been subjected to broader tests of generalizability ${ }^{4}$. More independent replication attempts of brain markers are needed, both to assess the current state of replicability and to spur the development of replicable predictive models.

Here, we conducted an independent replication of a previous report that response to cognitive-behavioral therapy (CBT) for social anxiety disorder (SAD) could be accurately predicted from baseline brain connectivity ${ }^{1}$. The authors reported that baseline amygdala-seeded functional connectivity explained $21 \%$ of the variance in treatment response, with incremental validity above and beyond a paper-and-pencil measure of baseline symptom

\section{(c) The Author(s) 2021}

(c) (i) Open Access This article is licensed under a Creative Commons Attribution 4.0 International License, which permits use, sharing, adaptation, distribution and reproduction c. in any medium or format, as long as you give appropriate credit to the original author(s) and the source, provide a link to the Creative Commons license, and indicate if changes were made. The images or other third party material in this article are included in the article's Creative Commons license, unless indicated otherwise in a credit line to the material. If material is not included in the article's Creative Commons license and your intended use is not permitted by statutory regulation or exceeds the permitted use, you will need to obtain permission directly from the copyright holder. To view a copy of this license, visit http://creativecommons.org/licenses/by/4.0/. 
severity. Treatment response was predicted by a linear combination of positive amygdala connectivity with a subgenual cingulate/caudate/putamen cluster, negative amygdala connectivity with bilateral central sulcus clusters, and negative amygdala connectivity with a right temporal-occipital cluster. We conducted our replication attempt in an existing dataset ${ }^{22,23}$ which was similar to the original study in key respects, with baseline resting-state fMRI collected on SAD patients prior to CBT treatment, though some differences in sample characteristics and treatment implementations are noted, described further below.

Alterations in amygdala function and structure have been one of the most reliable findings that distinguish SAD patients from healthy controls, including greater amygdala responses to threatening social stimuli and altered profiles of resting connectivity ${ }^{24,25}$. Several reports have also highlighted functional or structural properties of the amygdala that are impacted by CBT, often correlating with improvements in clinical outcomes ${ }^{22,26-28}$. And, paralleling the Whitfield-Gabrielli et al. findings studied here, two other studies have reported that different features of amygdala function predict SAD treatment response to $\mathrm{CBT}^{29,30}$. Yet, to our knowledge, none of these studies have been directly subjected to independent replication attempts. Collectively, this has generated an important set of findings with a coherent, consistent focus on the amygdala-but with unknown replicability.

Independent replication attempts of precisely specified models are needed to advance the field toward clinical and societal applications. Scientific collaborations will support this effort, leading to a cumulative science creating research products with both clinical and scientific applications. Here, we test the precise model specified in the original report. In addition, we also tested a variant of the original model, to better understand how amygdala connectivity predicts treatment response in the replication dataset.

\section{Methods}

The replication and original datasets were similar in key respects, with a comparison provided in Table 1 and discussed in greater detail below. The replication dataset has been previously described in studies examining the effects of cognitive-behavioral therapy (CBT) on brain function (NCT00380731) ${ }^{22}$. No analyses aiming to predict treatment response from baseline imaging have been previously reported in the replication dataset.

\section{Participants}

Participants in the replication dataset were recruited through referrals and web listings between 2007 and 2010. Participants were required to have a principal diagnosis of a $\mathrm{SAD}$, with no current pharmacotherapy or psychotherapy.
SAD was assessed by a diagnostic interview conducted by Ph.D.-trained clinical psychologists using the Anxiety Disorders Interview Schedule for DSM-IV (ADIS- IV) ${ }^{31}$, which has strong inter-rater reliability ${ }^{32}$. Diagnostic criteria for SAD were defined as greater than moderate fear in five or more distinct social situations, LSAS score of $\geq 60$, and a clinician-assigned ADIS-IV clinical severity rating of 4 or greater ( $0-8$ scale) for $\mathrm{SAD}$. This was similar to the original study, which used either the SCID or ADIS for DSM-IV to confirm SAD diagnosis and also required LSAS score $\geq 60$ (Table 1).

Exclusion criteria included current or past CBT, history of neurological disorders, or meeting diagnostic criteria for any current psychiatric condition other than generalized anxiety disorder, agoraphobia without panic attacks, specific phobia, panic disorder, or dysthymia. This was similar but not identical to the original study, which allowed the presence of comorbid mood disorders if SAD was judged to be the predominant disorder.

We required participants to be unmedicated for at least 1 year prior to the baseline scan; the original study required a period of at least two weeks without psychiatric medications. We also required participants to be righthanded based on the Edinburgh Handedness Inventory ${ }^{33}$ and to pass an MRI safety screen. The original study did not require right-handedness (though most participants in the sample were right-handed). Participants in the replication dataset provided informed consent in compliance with the Stanford University Institutional Review Board.

\section{Procedures}

After passing a telephone screen and an in-person eligibility session, participants completed a baseline assessment session including fMRI. Participants were then randomly assigned using biased coin randomization to either individual CBT $(n=38)$ or to a waitlist (WL) control group $(n=37)$ who were subsequently offered CBT. Pre-randomization resting-state fMRI data were collected on $n=53$ subjects of these subjects ( $n=30$ CBT immediate, $n=23$ CBT post-waitlist). All participants who completed at least 12 of 16 CBT sessions were included in analyses, with their last available measure of social anxiety symptom severity used in analyses (last observation carried forward).

CBT included 16 individual weekly sessions over 4 months. It was delivered using Managing Social Anxiety: A Cognitive-Behavioral Therapy Approach, a manualized treatment protocol that included a therapist guide $^{34}$ and a client workbook ${ }^{35}$. All four study therapists had demonstrated proficiency in CBT with training cases prior to treating study patients. Therapists were trained and supervised by Richard Heimberg, PhD, an expert in CBT for SAD and one of the developers of the 
Table 1 Comparison of original and replication datasets.

\begin{tabular}{|c|c|c|}
\hline & Original report & Replication dataset \\
\hline Primary reference & Whitfield-Gabrieli et al. ${ }^{1}$ & Goldin et al., 2012, 2013 \\
\hline Patient population & Social anxiety disorder & Social anxiety disorder \\
\hline Sample size & 38 & 42 \\
\hline Age, years & $M=29.2$, range $18-49$ & $M=34.5, \mathrm{SD}=8.89$, range $21-53$ \\
\hline Gender & $63 \%$ male & $45.2 \%$ male \\
\hline Race/Ethnicity & Not reported & $\begin{array}{l}\text { 54.8\% Caucasian, 26.2\% Asian, } \\
\text { 7.2\% Filipino/Pacific Islander, } \\
\text { 7.1\% Hispanic/Latinx, 2.4\% Black, 2.4\% more than one }\end{array}$ \\
\hline Education, years & Not reported & $M=17.0, \mathrm{SD}=2.20$, range $13-21$ \\
\hline Treatment & 12 weeks of group CBT & 16 weeks of individual CBT \\
\hline $\begin{array}{l}\text { SAD definition and inclusion } \\
\text { criteria }\end{array}$ & $\begin{array}{l}\text { SAD diagnosis confirmed using either the SCID or ADIS } \\
\text { for DSM-IV; LSAS } \geq 60\end{array}$ & $\begin{array}{l}\text { SAD diagnosis confirmed using the ADIS for DSM-IV, with } \geq 4 \\
\text { ADIS clinical severity rating; LSAS } \geq 60\end{array}$ \\
\hline Primary clinical outcome & LSAS & $\begin{array}{l}\text { LSAS (baseline } M=82.0[S D=17.9] \text {; post-CBT } M=51.3 \\
{[24.2] \text { ) }}\end{array}$ \\
\hline Medication status & Unmedicated for at least 2 weeks prior to baseline fMRI & Unmedicated for at least 1 year prior to baseline fMRI \\
\hline Psychiatric comorbidities & $\begin{array}{l}\text { Other mood or anxiety disorders permitted if SAD } \\
\text { judged to be the predominant disorder; other } \\
\text { psychiatric conditions excluded. }\end{array}$ & $\begin{array}{l}\text { GAD, agoraphobia, specific phobia, panic disorder, and } \\
\text { dysthymia permitted; other psychiatric conditions excluded. } \\
11.9 \% \text { with current Axis I comorbidity; } 26.2 \% \text { with past Axis I } \\
\text { diagnosis. }\end{array}$ \\
\hline $\begin{array}{l}\text { Age of SAD onset, mean } \\
(\mathrm{SD}) \text {, years }\end{array}$ & 12.2 & $14.26(8.32)$ \\
\hline $\begin{array}{l}\text { SAD duration, mean } \\
(\mathrm{SD}) \text {, years }\end{array}$ & 17.4 & $20.45(12.91)$ \\
\hline Scanner & 3 T Siemens Trio Tim & GE 3-T Signa \\
\hline Headcoil & Siemens 32-channel & Quadrature coil \\
\hline Scan parameters & $6 \mathrm{~min}, \mathrm{TR}=6 \mathrm{~s}, 2 \times 2 \times 2 \mathrm{~mm}$ resolution, gradient echo & $5 \mathrm{~min}, \mathrm{TR}=1.5 \mathrm{~s}, 3.4 \times 3.4 \times 4.5 \mathrm{~mm}$ resolution, spin echo \\
\hline Task & Fixate on crosshairs & Fixate on crosshairs \\
\hline Recruitment region & Boston area & San Francisco Bay Area \\
\hline
\end{tabular}

CBT cognitive behavioral therapy, LSAS Liebowitz Social Anxiety Scale, GAD generalized anxiety disorder, SAD social anxiety disorder.

treatment protocol used here. All four therapists demonstrated adherence to treatment protocols, as verified by independent raters, as detailed in the original report $^{23}$.

Participants in the WL group initiated individual CBT 19 weeks after the baseline brain scan. We included these participants in analyses as it is unknown whether the biomarker tested here predicted the effects of CBT specifically, placebo effects, the natural history of symptoms, or some combination of these factors since the original report did not include a control group. Given the likelihood that the biomarker predicted a combination of these factors ${ }^{36}$, we reasoned that the post-waitlist CBT participants also offered a fair test for the biomarker while allowing us a larger sample size. Thus, considering dropout, the final sample consisted of $n=42 \quad(n=25$ CBT-immediate, $n=17$ CBT post-waitlist). For completeness, we also conducted the replication in the CBTimmediate group only.

\section{Measures}

The Liebowitz Social Anxiety Scale (LSAS) self-report form $^{37,38}$ was the primary outcome, as in the original report. LSAS scores range from 0 to144, with traditional cutoffs for mild, moderate, and severe symptom severity at 30, 50, and 90. Treatment response was defined as the pre-to-post-treatment change in LSAS. The LSAS has excellent reliability and construct validity ${ }^{39}$, and its internal consistency was excellent in this study (Cronbach's alpha $=0.91)$. 


\section{Data acquisition}

Imaging was performed on a GE 3-T Signa magnet with a T2*-weighted gradient-echo spiral-in/spiral-out pulse sequence and a custom-built quadrature "dome" elliptical birdcage head coil (GE Healthcare, Milwaukee, Wisconsin). Participants completed a 5-min resting-state functional run while fixating on cross-hair visual stimuli. 200 functional volumes were obtained from 22 sequential axial slices (repetition time $=1.5 \mathrm{~s}$, echo time $=30 \mathrm{~ms}$, flip angle $=60^{\circ}$, field of view $=22 \mathrm{~cm}$, matrix $=64 \times 64$, singleshot, resolution $=3.438 \mathrm{~mm}^{2} \times 4.5 \mathrm{~mm}$ ). Threedimensional high-resolution anatomical scans were acquired using fast spin-echo spoiled gradient recall $(0.85942 \times 1.5 \mathrm{~mm}$; field of view $=22 \mathrm{~cm}$, frequency encoding $=256$ ).

In the original report, a 6-min resting-state scan was collected on a $3 \mathrm{~T}$ Siemen's Trio Tim scanner was collected while participants fixated on a cross-hair (T2* weighted gradient echo repetition time/echo time/Flip angle $=6000 \mathrm{~ms} / 30 \mathrm{~ms} / 90^{\circ}, 67$ contiguous interleaved oblique slices, voxel size: $2.0 \mathrm{~mm}^{3}$ ).

\section{Preprocessing of $\mathrm{fMRI}$ data}

Preprocessing followed the same procedures and tools described in the original report, using the Conn toolbox ${ }^{40}$ wrapping SPM12 for preprocessing routines. We performed slice time correction, motion estimation and realignment, normalization to MNI305 space, and spatial smoothing with an $8 \mathrm{~mm}$ FWHM Gaussian filter. GLM regression was used to remove the influence of with the following nuisance covariates: six head motion parameters and their first-order temporal derivatives, the first three components of white matter and CSF tissue compartments, and spike regressors identifying volumes flagged as outliers by the Artifact Detection Tool ${ }^{40}$ (an image was defined as an outlier if the head displacement in any direction was $\geq 0.5 \mathrm{~mm}$ from the previous frame, or if the global mean image intensity $\geq 3$ standard deviations above the mean image intensity for the scan). The resulting residual BOLD time-series were then band-pass filtered $(0.01 \mathrm{~Hz}<\mathrm{f}<0.10 \mathrm{~Hz})$. The replication dataset had acceptable levels of head motion, quantified as framewise displacement $(\mathrm{FD}) \quad\left(M_{\mathrm{FD}}=0.13 \mathrm{~mm}, \quad\right.$ Median $_{\mathrm{FD}}=$ $\left.0.06 \mathrm{~mm}, \mathrm{SD}_{\mathrm{FD}}=0.13 \mathrm{~mm}\right)$.

\section{Comparison of the original and replication datasets}

The original and replication datasets were similar in key respects. They also differed in some characteristics, such as group vs. individual CBT, scan acquisition parameters, the geographic region from which patients were recruited, and other characteristics (see Table 1 for a full comparison).

In addition, the original study randomized subjects to receive either $\mathrm{D}$-cycloserine or placebo in conjunction with CBT. No drug vs. placebo differences emerged, and the authors collapsed across conditions in their analyses. The treatment in the original study might thus be considered "placebo-enhanced" CBT. While meta-analyses show a placebo effect of $g=0.39$ in $\mathrm{SAD}^{41}$, it is unknown to what extent this effect is additive, interacting, or fully overlapping with psychotherapy effects ${ }^{36}$, and so it cannot be determined to what extent drug administration impacted outcomes in the original study. Importantly, randomization to D-cycloserine or placebo occurred after the baseline imaging session in the original study, so there were no drug effects on functional connectivity. No medication was administered in the replication study, a potentially important difference between the two datasets.

\section{Model specification}

Details regarding the predictive model specification and the cluster masks were provided in personal communications with the original authors. Mean connectivity between the amygdala and the single positive connectivity cluster was averaged with mean amygdala connectivity with the three negative connectivity clusters. This formed a single connectivity term, which was Fisher-transformed and Z-scored across subjects. This connectivity term was then submitted to a GLM along with baseline symptom severity (LSAS) to predict treatment response, operationalized as the pre-to-post-treatment change in LSAS ( $\triangle$ LSAS). The final predictive model derived in the original report was:

$\Delta$ LSAS $=0.6194 *$ baseline_LSAS $+8.6290 *$ amyg_conn - 9.9763 (1), where amyg conn refers to the amygdala connectivity term. The original report compared predictions from (1) to a compact model including only baseline LSAS and an intercept term as predictors.

Precisely specified models are necessary for a replicable, cumulative science. We thus sought to apply this exact model to our data. We made one modification, dropping the intercept term from the model (and mean-centering all model variables in the replication dataset). This removed intercept effects in treatment response, as these are reasonably expected to vary from study to study.

The original model included dependence between a predictor (baseline LSAS) and the outcome (change in LSAS from baseline to post-treatment). Thus, we first tested whether the covariance between baseline and change in LSAS was similar across the original and replication datasets. We compared model predictions derived from the optimal OLS model fit in the replication data to predictions using the parameter estimate from the original model (i.e., $\beta_{\text {baseline_LSAS }}=0.6194$ ).

In addition, to explore a more flexible form of replication, we conducted a GLM estimating new parameter estimates for the original model terms. Here, we tested whether the amygdala connectivity term significantly 
predicted treatment response, controlling for baseline LSAS.

The original report also reported successful prediction of treatment response from multivoxel pattern analyses (MVPA) of resting fMRI and from diffusion tensor imaging (DTI). We were unable to apply the MVPA-derived model to our data: One of the predictive clusters in the MVPA model was an inferior cerebellar region for which we had incomplete coverage in the replication dataset. Similarly, DTI was not collected in the replication dataset.

\section{Model assessment}

We assessed performance by comparing predictions from the full model to a compact model including only the baseline LSAS term. Prediction performance was measured using two metrics, following recent recommendations ${ }^{3,42}$.

First, we computed an absolute measure of improvement in prediction, normalized mean square error (NMSE). We computed the mean squared error (MSE) between the observed data and the predictions from the full model, divided by MSE between the observed data and the predictions from the compact model. We then

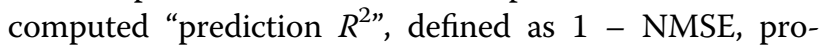
viding the proportional reduction in error for the full model vs. the compact model ${ }^{42}$.

The second metric we used was a model-based $R^{2}$. We computed the squared Pearson correlation between the observed data and the full model predictions, as compared to the squared correlation between the observed data and the compact model predictions. Correlation provides a scale-free measure of predictive accuracy, which may be appropriate for testing the model in independent data, though in doing so it provides increased model flexibility. Squaring the correlation coefficient provided a modelbased estimate of $R^{2}$.

Statistical significance of prediction and model-based $R^{2}$ was assessed by repeatedly permuting the amygdala connectivity term across subjects, generating a null distribution, and comparing the unpermuted result to the 95th percentile of the null distribution (10,000 permutations). Analyses were conducted using the CanlabCore toolbox, a freely available MATLAB $\odot$ toolbox for flexible neuroimaging analyses: https://github.com/canlab/CanlabCore. Data and code for analyses are publicly available at https:// github.com/yonestar/WhitfieldGabrieli2015_replication.

\section{Results}

\section{Predicting treatment response from baseline symptoms}

In the original report, the model including only baseline social anxiety symptom scores ("compact model") predicted $12 \%$ of the variance in pre-to-post-treatment change scores: Higher pre-treatment scores predicted greater pre-to-post-treatment symptom reductions, owing both to regression to the mean and to dependence between the two variables.

In the replication dataset, baseline social anxiety symptoms explained $20 \%$ of the variance in pre-to-posttreatment change scores. The OLS parameter estimate for baseline symptom scores in the replication dataset was $\beta_{\text {baseline_LSAS }}=0.66$, numerically similar to the parameter estimate from the original model $\left(\beta_{\text {baseline_LSAS }}=0.62\right)$. We confirmed that the OLS parameter estimate provided only a very small improvement in prediction over the original parameter estimate $\left(<1 \%\right.$, prediction $\left.R^{2}=0.0012\right)$. Since both the OLS-derived and the original parameter estimate for baseline social anxiety symptoms performed very similarly in predicting treatment response in the replication data, we used the original parameter estimate in the replication analyses to provide the most direct test of model replication.

\section{Predicting treatment response from amygdala connectivity}

In the original report, the addition of the amygdala connectivity term led to a total $33 \%$ variance explained, a substantial increase of $21 \%$ over the variance explained by baseline social anxiety symptoms alone. In the replication dataset, the amygdala connectivity term explained an additional $2 \%$ of the variance (Fig. $1 C$; prediction $R^{2}=$ 0.016 , model-based $R^{2}=0.020$ ). This improvement in prediction was marginally significant, model-based $p=$ 0.097 and prediction $R^{2} p=0.101$, rendering it unclear whether the small increase in predictive power afforded by amygdala connectivity was due to chance.

Visual inspection of the data confirmed that findings were not driven by outliers (Fig. 1D). We confirmed that all three terms in the model were approximately normally distributed, Anderson-Darling test, $p \mathrm{~s}>0.4$.

\section{Predicting treatment response with increased model flexibility}

To provide a more flexible test of model replication, we estimated a GLM including the same model terms as in the original report. Controlling for baseline LSAS, the amygdala connectivity term did not significantly predict treatment response, $\beta_{\text {amyg_conn }}=4.78, t(40)=1.31, p=0.20$.

\section{Testing model predictions in CBT-immediate subset}

In the subset of 25 participants who received CBT immediately after the baseline neuroimaging session (CBT-immediate), the model did not predict treatment response. In this subset, predictions from baseline LSAS alone (compact model), the OLS estimate for $\beta_{\text {baseline_LSAS }}=0.65$, highly similar to original model value $\left(\beta_{\text {baseline_LSAS }}=0.62\right)$. We thus used the original model

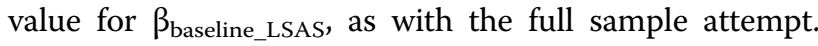
Relative to the compact model, the full model including 

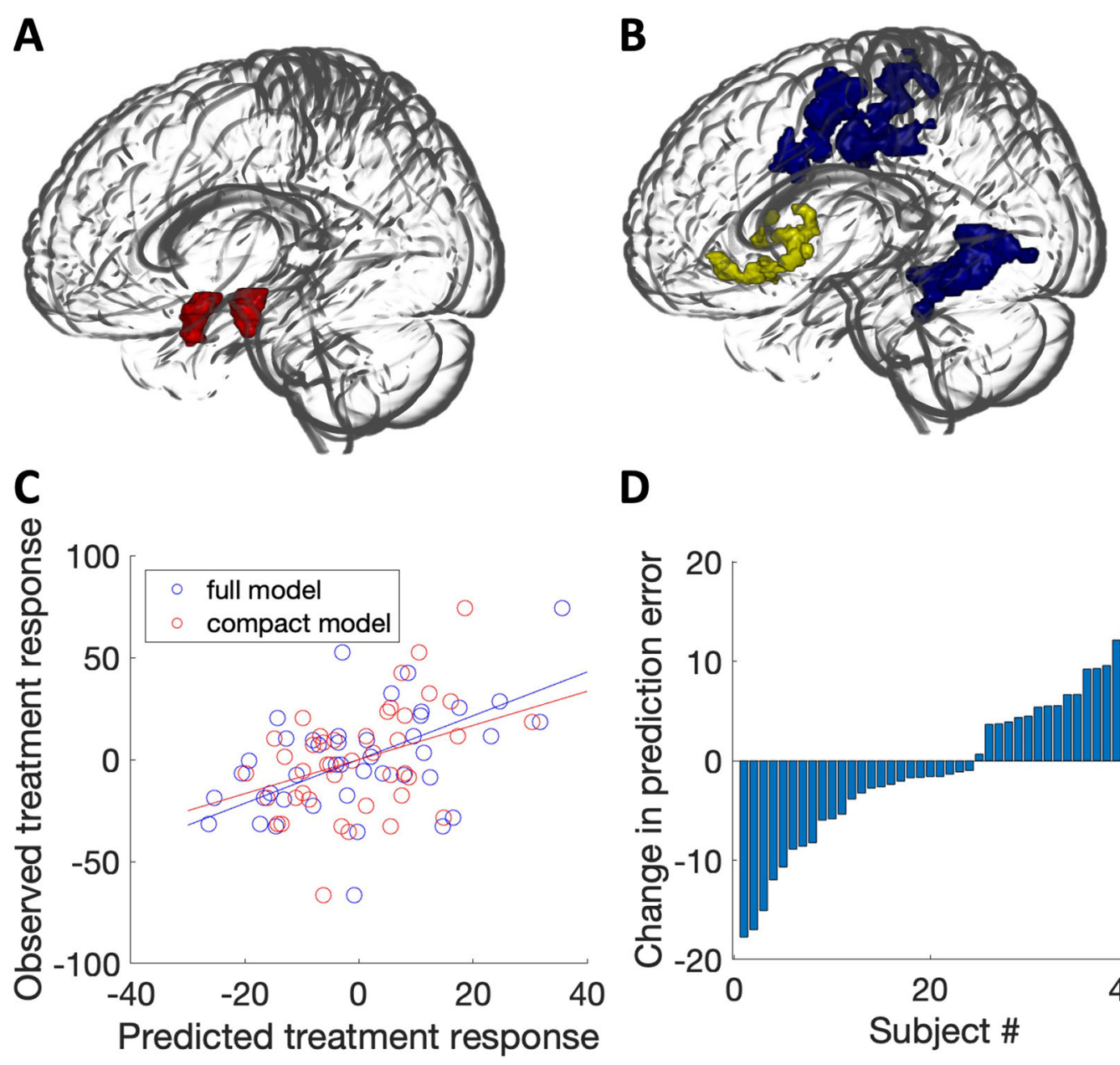

D

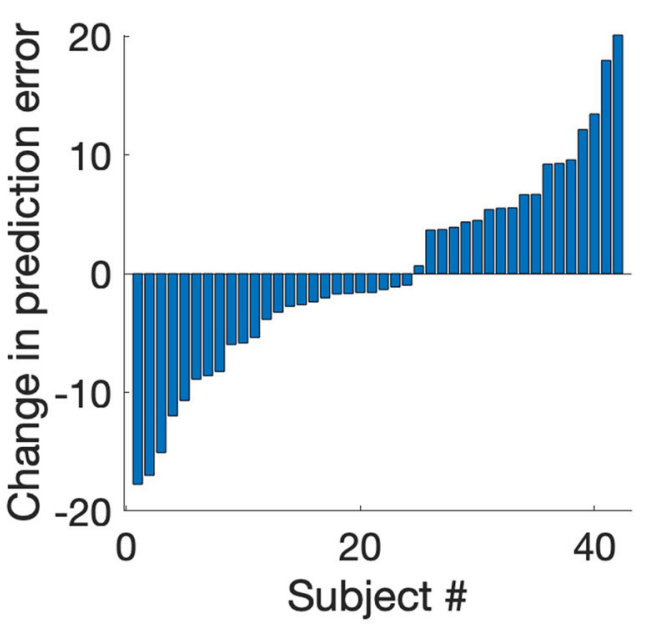

Fig. 1 The predictive model and independent replication results. A The seed region for the connectivity-based predictive model: bilateral, anatomically defined amygdala (red). B Treatment response was predicted by a linear combination of greater amygdala connectivity with a subgenual cingulate/caudate/putamen cluster (yellow) and reduced amygdala connectivity with bilateral central sulcus clusters and right temporaloccipital clusters (blue). C Predicted treatment response for the compact model (including only baseline symptom severity, red points) vs. the full model (also including amygdala connectivity, blue points). Successful replication would be indicated by an improved prediction for the full model vs. the compact model. D Changes in prediction errors for the full vs. compact model for each subject. The inclusion of amygdala connectivity slightly improved model predictions (increase of $2 \%$ variance explained), with marginal statistical significance. Brain images were created using MRICroGL.

amygdala connectivity did not improve the prediction of treatment response, prediction $R^{2}=-0.019, p=0.18$, model-based $R^{2}=0.007, p=0.20$. Fitting the predictive model in this subset of participants, the amygdala connectivity term did not significantly predict treatment response controlling for baseline symptom severity, $\beta_{\text {amyg_conn }}=3.84, t(40)=0.81, p=0.43$.

\section{Discussion}

We conducted an independent replication of a model previously shown to predict response to CBT for SAD from resting-state amygdala-seeded fMRI connectivity ${ }^{1}$. We tested the precise model developed in the original report. This model provided a small improvement in the prediction of treatment response. It explained an additional $2 \%$ of the variance beyond baseline symptom severity, which attained marginal statistical significance and was approximately $1 / 10$ th of the effect size in the original report. A more flexible test of model replication, which used the amygdala connectivity target regions from the original report and estimated model parameters on the replication dataset, found no significant prediction of treatment response. Overall, our results support the hypothesis that some features of the amygdala might predict treatment response in SAD, but that more strongly predictive models must be developed for scientific and clinical applications.

The original and replication datasets were similar in many characteristics, and at the same time, differences between them in patient characteristics, treatment 
implementations, and fMRI parameters may have contributed to the current findings. The samples were similar in SAD age of onset, SAD duration, and inclusion/ exclusion criteria (Table 1). And yet, different symptom profiles within a diagnostic category, as well as different patterns of psychiatric comorbidities, have been associated with functional connectivity differences ${ }^{43-46}$. CBT implementations also varied between the two studies, including individual vs. group CBT and the "placebo enhancement" of CBT in the original dataset, another important difference. And scanner hardware and acquisition parameters differed between the two studies. While this was not an exact replication, we believe that predictive markers will need to be robust to reasonable variations in many of these characteristics for practical scientific and clinical applications.

We focus our discussion here on five factors of interest that likely influenced the current findings and can also guide future attempts to develop generalizable predictive biomarkers. These include: (a) sample size, (b) reliability of fMRI connectivity measures, (c) dimensional rather than categorical models of dysfunctional phenotypes, (d) incremental validity and "broad" data, and (e) data sharing and multi-team collaborations.

First, both the original and replication datasets were underpowered due to relatively small sample sizes. With approximately $N=40$ in each dataset, these studies provide only $50 \%$ power to detect a medium effect $(\rho=0.3)$. Stable estimates for correlation coefficients describing medium effects require $N=100-250$, depending on the desired confidence interval ${ }^{47}$. The original findings $-21 \%$ variance explained by amygdala connectivity in a sample of size $N=38$-are accompanied by wide $95 \%$ confidence intervals ranging from 2.7 to $46 \%$.

A second factor is the reliability of fMRI connectivity measures. In the original and replication datasets, scans were 6 and $5 \mathrm{~min}$, lengths unlikely to yield reliable seedbased connectivity estimates ${ }^{48,49}$. Recent work with highly sampled subjects has found that 30-40 min of motioncensored data per subject provides reliable connectivity estimates $^{50,51}$, with perhaps twice this scan length needed for subcortical structures ${ }^{52}$. In addition to longer scans, advances in acquisition and analysis technologies, such as multi-echo fMRI $^{53}$ and custom head molds ${ }^{54}$, improve data quality. Analytic approaches can also improve reliability, with multivariate markers likely providing a broader base of support relative to single regions of interest ${ }^{6,55}$. In addition, methods for improved inter-subject alignment $^{56,57}$ and systematic approaches for developing biomarker pipelines ${ }^{58,59}$ will improve performance. Finally, spatial resolution is an additional consideration, especially for smaller structures like the amygdala, and effective resolution will be determined by the smoothing kernel applied.
Third, both and the original and replication studies considered the SAD diagnosis as a categorical indicator of a shared dysfunction. However, it is well known that psychiatric patient populations are heterogenous: a shared diagnosis does not require shared mechanisms or even shared symptoms ${ }^{60,61}$. Predictive markers focused on patient subtypes or dimensional descriptions of dysfunction may relate more strongly to biology, providing greater traction for predictive biomarkers ${ }^{46}$. Several dimensional models have been recently proposed, including the NIH research domain criteria ${ }^{62}$ and the Hierarchical Taxonomy of Psychopathology ${ }^{63}$, and some recent studies have adopted a dimensional subtyping approach with encouraging results ${ }^{43,64,65}$.

A fourth factor is an incremental validity and "broad" data ${ }^{66}$. Biomarkers will be most useful if they have predictive utility above and beyond measures that are cheaper and easier to acquire. Demonstration of incremental validity requires collecting a "broad" set of measures from multiple channels (ecological momentary assessment, behavior, physiology, smartphone usage data, etc.) to compare with neuroimaging-based prediction. Critically, "broad" data will advance understanding of how environmental influences mediate the relationship between biology and phenotypic measures of dysfunction (e.g., biomarkers predictions may only hold in a particular socioeconomic group or only for subjects with high inflammation ${ }^{67}$ ).

Fifth, for a cumulative scientific process to yield generalizable predictive biomarkers, data sharing and collaborative replication efforts are needed, such as the current effort $^{4,17,19,21}$. Software tools, data sharing platforms, and reproducibility pipelines are needed to support this effort $^{21,68,69}$. We believe it is important to replicate precisely defined models ${ }^{70}$. For example, the amygdala is a sensible focus point for predictive models relating to SAD, given the large body of research indicating amygdala alterations in SAD and other anxiety disorders ${ }^{24,25}$, and with several studies reporting that features of amygdala function predict CBT response in SAD patients ${ }^{1,29,30}$. However, the particular predictive amygdala features have varied widely from study to study, often with little overlap. It is not enough to know that "something about the amygdala" predicts treatment response-precisely specified models are needed. An aggregation of all relevant datasets with resting-state fMRI prior to CBT in SAD will provide a strong foundation for collaborative efforts to develop predictive models. While the specific model tested here may not explain sufficient variance in independent datasets for scientific or clinical applications, we believe our results can support a continued focus on amygdala connectivity in developing improved predictive models.

There are many exciting opportunities as well as challenges in the effort toward building generalizable 
predictive biomarkers. We believe collaborative independent replication attempts, such as the one undertaken here, will play a critical role in this process, regardless of replication results.

\section{Acknowledgements}

Funded by NIH R01 R01DA035484 (PI Wager), NIH R01 MH76074 (PI Gross), and NIH NCATS Grant \#TL1-TR-002386 (PI Ashar). Data and code are publicly available at https://github.com/yonestar/WhitfieldGabrieli2015_replication. We thank the authors of Whitfield-Gabrielli et al., 2015 for their assistance in conducting this replication, and we thank the anonymous reviewers for their feedback on an earlier version of this manuscript.

\section{Author details}

${ }^{1}$ Department of Psychiatry, Weill Cornell Medicine, New York, NY, USA. ${ }^{2}$ Department of Psychology and Neuroscience, University of Colorado Boulder, Boulder, CO, USA. ${ }^{3}$ Betty Irene Moore School of Nursing, University of California, Davis, Davis, CA, USA. ${ }^{4}$ Department of Psychology, Stanford University, Palo Alto, CA, USA. ${ }^{5}$ Psychological and Brain Sciences Department, Dartmouth College, Hanover, NH, USA

\section{Author contributions}

Y.A., T.W., P.G., and J.G. conceptualized the study. Y.A. conducted the analyses and drafted the manuscript. J.C. assisted with data curation and analyses. T.W. supervised analyses. J.G. and P.G. provided the data for analyses. F.G. along with all other authors contributed to the interpretation of the results and to revisions of the manuscript.

\section{Conflict of interest}

The authors declare no competing interests.

\section{Publisher's note}

Springer Nature remains neutral with regard to jurisdictional claims in published maps and institutional affiliations.

Received: 3 March 2021 Revised: 18 March 2021 Accepted: 7 April 2021 Published online: 01 May 2021

\section{References}

1. Whitfield-Gabrieli, S. et al. Brain connectomics predict response to treatment in social anxiety disorder. Mol. Psychiatry https://doi.org/10.1038/mp.2015.109 (2015).

2. Gabrieli, J. D. E., Ghosh, S. S. \& Whitfield-Gabrieli, S. Prediction as a humanitarian and pragmatic contribution from human cognitive neuroscience. Neuron $\mathbf{8 5}$, 11-26 (2015).

3. Poldrack, R. A., Huckins, G. \& Varoquaux, G. Establishment of best practices for evidence for prediction: a review. JAMA Psychiatry 77, 534-540 (2019).

4. Woo, C.-W., Chang, L. J., Lindquist, M. A. \& Wager, T. D. Building better biomarkers: brain models in translational neuroimaging. Nat. Neurosci. Rev. 20, 365-377 (2017).

5. Davis, K. D. et al. Discovery and validation of biomarkers to aid the development of safe and effective pain therapeutics: challenges and opportunities. Nat. Rev. Neurol. https:/doi.org/10.1038/s41582-020-0362-2 (2020).

6. Kragel, P. A., Han, X., Kraynak, T., Gianaros, P. J. \& Wager, T. D. fMRI can be highly reliable, but it depends on what you measure. Psychol. Sci. https:/journals. sagepub.com/doi/abs/10.1177/0956797621989730?journalCode=pssa (2021).

7. Open Science Collaboration. Estimating the reproducibility of psychological science. Science 349, aac4716-aac4716 (2015).

8. Tackett, J. L., Brandes, C. M., King, K. M. \& Markon, K. E. Psychology's Replication Crisis and Clinical Psychological Science. Annu. Rev. Clin. Psychol. 15, 579-604 (2019).

9. Fox, A. S., Lapate, R. C., Davidson, R. J. \& Shackman, A. J. Epilogue: The Nature of Emotion. A Research Agenda for the 21st century. The Nature of Emotion. Fundamental Questions (2018).

10. Pankevich, D. E., Altevogt, B. M., Dunlop, J., Gage, F. H. \& Hyman, S. E. Improving and accelerating drug development for nervous system disorders. Neuron $\mathbf{8 4}$, 546-553 (2014).
11. Prinz, F., Schlange, T. \& Asadullah, K. Believe it or not: how much can we rely on published data on potential drug targets? Nat. Rev. Drug Discov. 10, 712-713 (2011).

12. Baker, M. \& Dolgin, E. Cancer reproducibility project releases first results. Nature 541, 269-270 (2017).

13. Button, K. S. et al. Power failure: why small sample size undermines the reliability of neuroscience. Nat. Rev. Neurosci. 14, 365-376 (2013).

14. Ioannidis, J. P. A. Why most published research findings are false. PLoS Med. $\mathbf{2}$, 2-8 (2005).

15. Camerer, C. F. et al. Evaluating the replicability of social science experiments in Nature and Science between 2010 and 2015. Nat. Hum. Behav. https:/doi.org/ 10.1038/s41562-018-0399-z (2018)

16. Botvinik-Nezer, R. et al. Variability in the analysis of a single neuroimaging dataset by many teams. Nature $\mathbf{5 8 2}, 84-88$ (2020).

17. Poldrack, R. A. et al. Scanning the horizon: Towards transparent and reproducible neuroimaging research. Nat. Rev. Neurosci. 18, 115-126 (2017).

18. Protzko, J. et al. High replicability of newly-discovered social-behavioral findings is achievable. PsyArXiv 1-27 (2020).

19. Munafò, M. R. et al. A manifesto for reproducible science. Nat. Hum. Behav. 1, 1-9 (2017).

20. Nelson, L. D., Simmons, J. \& Simonsohn, U. Psychology's Renaissance. Annu. Rev. Psychol. 69, 511-534 (2018).

21. Nichols, T. E. et al. Best practices in data analysis and sharing in neuroimaging using MRl. Nat. Neurosci. 20, 299-303 (2017).

22. Goldin, P. R. et al. Impact of cognitive behavioral therapy for social anxiety disorder on the neural dynamics of cognitive reappraisal of negative selfbeliefs: randomized clinical trial. JAMA Psychiatry 70, 1048-1056 (2013).

23. Goldin, P. R. et al. Cognitive reappraisal self-efficacy mediates the effects of individual cognitive-behavioral therapy for social anxiety disorder. J. Consult. Clin. Psychol. 80, 1034-1040 (2012).

24. Freitas-Ferrari, M. C. et al. Neuroimaging in social anxiety disorder: a systematic review of the literature. Prog. Neuro-Psychopharmacol. Biol. Psychiatry 34, 565-580 (2010).

25. Damsa, C., Kosel, M. \& Moussally, J. Current status of brain imaging in anxiety disorders. Curr. Opin. Psychiatry 22, 96-110 (2009).

26. Goldin, P. R., Manber-Ball, T., Werner, K., Heimberg, R. \& Gross, J. J. Neural mechanisms of cognitive reappraisal of negative self-beliefs in social anxiety disorder. Biol. Psychiatry 66, 1091-1099 (2009).

27. Young, K. S. et al. Treatment for social anxiety disorder alters functional connectivity in emotion regulation neural circuitry. Psychiatry Res. Neuroimaging 261, 44-51 (2017).

28. Månsson, K. N. T. et al. Neuroplasticity in response to cognitive behavior therapy for social anxiety disorder. Transl. Psychiatry 6, e727 (2016).

29. Burklund, L. J., Torre, J. B., Lieberman, M. D., Taylor, S. E. \& Craske, M. G. Neura responses to social threat and predictors of cognitive behavioral therapy and acceptance and commitment therapy in social anxiety disorder. Psychiatry Res. Neuroimaging 261, 52-64 (2017).

30. Young, K. S. et al. Neural connectivity during affect labeling predicts treatment response to psychological therapies for social anxiety disorder. J. Affect. Disord. 242, 105-110 (2019).

31. DiNardo, P., Brown, T. \& Barlow, D. Anxiety Disorders Intenview Schedule for DSMN: Lifetime version (ADIS-IV-L). (Oxford University Press, 1994).

32. Brown, T. A., Di Nardo, P. A., Lehman, C. L. \& Campbell, L. A. Reliability of DSMIV anxiety and mood disorders: implications for the classification of emotional disorders. J. Abnorm. Psychol. 110, 49-58 (2001).

33. Oldfield, R. C. The assessment and analysis of handedness: the Edinburgh inventory. Neuropsychologia 9.1, 97-113 (1971).

34. Hope, D., Heimberg, R. \& Turk, C. Therapist Guide for Managing Social Anxiety: A Cognitive-Behavioral Therapy Approach. (Oxford University Press, 2006).

35. Hope, D., Heimberg, R., Juster, H. R. \& Turk, C. Managing Social Anxiety: A Cognitive-Behavioral Approach. (Oxford University Press, 2000).

36. Ashar, Y. K. Chang, L. J. \& Wager, T. D. Brain mechanisms of the placebo effect: an affective appraisal account. Annu. Rev. Clin. Psychol. 13, 73-98 (2017).

37. Fresco, D. M. et al. The Liebowitz Social Anxiety Scale: a comparison of the psychometric properties of self-report and clinician-administered formats. Psychol. Med. 31, 1025-1035 (2001).

38. Liebowitz, M. R. in Mod Trends Pharmacopsychiatry (ed. Klein, D. F.). https:// www.karger.com/Article/Abstract/414022 (Basel, Karger, 1987).

39. Baker, S. L., Heinrichs, N., Kim, H. J. \& Hofmann, S. G. The Liebowitz social anxiety scale as a self-report instrument: a preliminary psychometric analysis. Behav. Res. Ther. 40, 701-715 (2002). 
40. Whitfield-Gabrieli, S. \& Nieto-Castanon, A. Conn: a functional connectivity toolbox for correlated and anticorrelated brain networks. Brain Connect 2 125-141 (2012).

41. Sugarman, M. A., Kirsch, I. \& Huppert, J. D. Obsessive-compulsive disorder has a reduced placebo (and antidepressant) response compared to other anxiety disorders: a meta-analysis. J. Affect. Disord. 218, 217-226 (2017).

42. Scheinost, D. et al. Ten simple rules for predictive modeling of individual differences in neuroimaging. Neuroimage 193, 35-45 (2019).

43. Drysdale, A. T. et al. Resting state connectivity biomarkers define neurophysiological biotypes of depression. Nat. Med. 1-3 (2017).

44. Feczko, E. et al. The heterogeneity problem: approaches to identify psychiatric subtypes. Trends Cogn. Sci. 23, 584-601 (2019).

45. Vachon-Presseau, E. et al. Identification of traits and functional connectivitybased neuropsychotypes of chronic pain. PLOS Biol. https://doi.org/10.1101/ 421438 (2018)

46. Kapur, S., Phillips, A. G. \& Insel, T. R. Why has it taken so long for biological psychiatry to develop clinical tests and what to do about it? Mol. Psychiatry 17 1174-1179 (2012).

47. Schönbrodt, F. D. \& Perugini, M. At what sample size do correlations stabilize? J. Res. Pers. 47, 609-612 (2013)

48. Noble, S., Scheinost, D. \& Constable, R. T. A decade of test-retest reliability of functional connectivity: A systematic review and meta-analysis. Neuroimage 203, 116157 (2019).

49. Gratton, $\mathrm{C}$. et al. Defining individual-specific functional neuroanatomy for precision psychiatry. Biol. Psychiatry 88, 28-39 (2020).

50. Gordon, E. M. et al. Precision functional mapping of individual human brains. Neuron 95, 791-807.e7 (2017).

51. Seitzman, B. A. et al. Trait-like variants in human functional brain networks. Proc. Natl Acad. Sci. USA 116, 22851-22861 (2019).

52. Greene, D. J. et al. Integrative and network-specific connectivity of the basal ganglia and thalamus defined in individuals. Neuron 105, 742-758.e6 (2020).

53. Lynch, C. J. et al. Rapid precision functional mapping of individuals using multi-echo fMRI. Cell Rep. 33, 108540 (2020).

54. Power, J. D. et al. Customized head molds reduce motion during resting state fMRI scans. Neuroimage 189, 141-149 (2019).

55. Elliott, M. L. et al. What is the test-retest reliability of common task-functional MRI measures? New empirical evidence and a meta-analysis. Psychol. Sci. 31 792-806 (2020).
56. Haxby, J. V., Connolly, A. C. \& Guntupalli, J. S. Decoding neural representational spaces using multivariate pattern analysis. Annu. Rev. Neurosci. https:/doi.org/ 10.1146/annurev-neuro-062012-170325 (2014).

57. Du, Y. et al. NeuroMark: a fully automated ICA method to identify effective fMRI markers of brain disorders. https://www.sciencedirect.com/science/ article/pii/S2213158220302126 (2019).

58. Lee, J. J. et al. A neuroimaging biomarker for sustained experimental and clinical pain. Nat. Med. 27, 174-182 (2021).

59. Abraham, A. et al. Deriving reproducible biomarkers from multi-site restingstate data: An autism-based example. Neuroimage 147, 736-745 (2016).

60. Lilienfeld, S. O. The Research Domain Criteria (RDoC): an analysis of methodological and conceptual challenges. Behav. Res. Ther. 62, 129-139 (2014).

61. Olbert, C. M., Gala, G. J. \& Tupler, L. A. Quantifying heterogeneity attributable to polythetic diagnostic criteria: Theoretical framework and empirical application. J. Abnorm. Psychol. 123, 452-462 (2014).

62. Cuthbert, B. N. \& Kozak, M. J. Constructing constructs for psychopathology: The NIMH research domain criteria. J. Abnorm. Psychol. 122, 928-937 (2013).

63. Conway, C. C. et al. A hierarchical taxonomy of psychopathology can transform mental health research. Perspect. Psychol. Sci. 14, 419-436 (2019).

64. Grisanzio, K. A. et al. Transdiagnostic symptom clusters and associations with brain, behavior, and daily function in mood, anxiety, and trauma disorders. JAMA Psychiatry 75, 201-209 (2018).

65. Clementz, B. A. et al. Identification of distinct psychosis biotypes using brainbased biomarkers. Am. J. Psychiatry 173, 373-384 (2016).

66. Shackman, A. J. \& Fox, A. S. Getting serious about variation: Lessons for Clinical Neuroscience (A Commentary on The Myth of Optimality in Clinical Neuroscience'). Trends Cogn. Sci. 22, 368-369 (2018).

67. Holmes, A. J. \& Patrick, L. M. The Myth of Optimality in Clinical Neuroscience. Trends Cogn. Sci. 22, 241-257 (2018).

68. Kennedy, D. N. et al. Everything matters: The reproNim perspective on reproducible neuroimaging. Front. Neuroinform. 13, 1 (2019).

69. Boland, M. R., Karczewski, K. J. \& Tatonetti, N. P. Ten simple rules to enable multi-site collaborations through data sharing. PLoS Comput. Biol. 13, 1-12 (2017).

70. Lumian, D. S. \& McRae, K. Preregistered replication of "affective flexibility: evaluative processing goals shape amygdala activity". Psychol. Sci. 28, 1193-1200 (2017) 